\title{
TAU NEUTRINO COMPONENT TO TRITIUM BETA DECAY
}

\section{LDRD REPORT}

\author{
Neal J. Snyderman \\ University of California \\ Lawrence Livermore National Laboratory \\ Livermore, CA 94550
}

June 1995

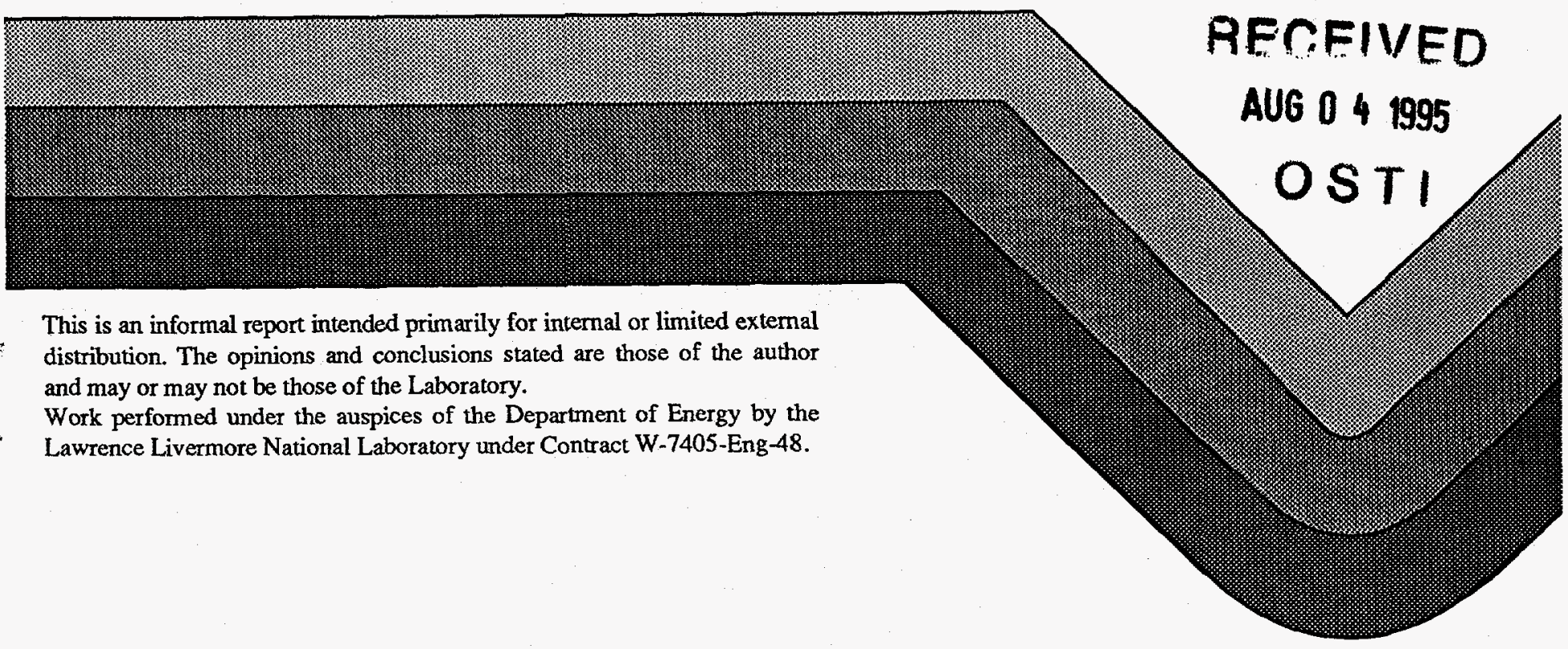

DISTRIBUTION OF THIS DOCUMENT IS UNLIMITEO 


\section{DISCLAIMER}

This report was prepared as an account of work sponsored by an agency of the United States Government. Neither the United States Government nor any agency thereof, nor any of their employees, make any warranty, express or implied, or assumes any legal liability or responsibility for the accuracy, completeness, or usefuiness of any information, apparatus, product, or process disclosed, or represents that its use would not infringe privately owned rights. Reference herein to any specific commercial product, process, or service by trade name, trademark, manufacturer, or otherwise does not necessarily constitute or imply its

endorsement, recommendation, or favoring by the United States Government or any agency thereof. The views and opinions of authors expressed herein do not necessarily state or reflect those of the United States Government or any agency thereof. 


\section{DISCLAIMER}

Portions of this document may be illegible in electronic image products. Images are produced from the best available original document. 


\title{
Tau Neutrino Component to Tritium Beta Decay \\ LDRD Report \\ Neal J. Snyderman \\ Lawrence Livermore National Laboratory \\ Livermore, CA 94550
}

\begin{abstract}
A framework is given for explaining anomalous results of neutrino mass experiments that measure the high energy electron spectrum of tritium $\beta$ decay. The experimental results have been fit to a negative neutrino mass square. We show that there is a consistent phenomenological interpretation due to a positive mass tau neutrino component of the $\beta$ decay spectrum, with strong near threshold final state interactions with the He nucleus. If this enhancement is due to new interactions between low energy tau neutrinos and nuclei, then the tritium $\beta$ decay experiments could be used as detectors for cosmic background tau neutrinos. The model predicts a distinctive spectrum shape that is consistent with a recent high statistics LLNL experiment. A fit to the experiment gives a tau neutrino mass of $23 \mathrm{eV}$. Tau neutrinos of this mass would dominate the mass of the universe. Requirements for a theoretical model are given, as well as models that realize different aspects of these requirements. While qualitatively successful, the theoretical models have such severe quantitative difficulties that the accuracy of the molecular physics of the $\mathrm{T}^{3}{ }^{3} \mathrm{He}$ ion, assumed in the analysis of the experimental data, is called into question.
\end{abstract}

There has been a persistent anomaly observed in the tritium $\beta$ decay spectrum [1]. These experiments measure electrons emitted near the high energy end point, $\mathrm{E}_{0}$. Fermi used measurements of $\mathrm{E}_{0}$ to determine the mass differences of nuclei. If , however, $\mathrm{E}_{0}$ is accurately known from mass differences, then for massive neutrinos a cutoff in the spectrum is expected for electron energies approaching $\mathrm{E}_{0}-\mathrm{m}_{\mathrm{v}}$. Instead, the experiments [1] have detected more electrons, relative to those predicted for massless neutrinos, just below the high energy end point. For this reason, the results of these experiments have been quoted in terms of limits for $m_{V}$ arising from a fit with negative neutrino mass squared. Fits to the end point from a recent high statistics experiment [2] are consistent, within $\sim 1 \mathrm{eV}$ experimental errors, with $\mathrm{E}_{0}$ as determined from Ion Cyclotron Resonance 
measurement of the ${ }^{\mathrm{T}}-{ }^{3} \mathrm{He}$ mass difference [3], that is, consistent with a massless neutrino. However, there is a distinct enhancement of the spectrum near, but ending at, $\mathrm{E}_{0}-\mathrm{m}_{\mathrm{V}}$, with $\mathrm{m}_{\mathrm{V}} \sim 23 \mathrm{eV}$ [2]. We suggest that the combined observations of the high energy electron endpoint, $\mathrm{E}_{0}$, but with an enhanced spectrum $20-30 \mathrm{eV}$ below the endpoint, are consistent with a massive electron neutrino that is a mixture of three massive neutrino species, with a small weight for a heavy component of mass $\mathrm{m}_{\mathrm{v}} 3 \sim 23$ $\mathrm{eV}$. (Since the tau neutrino is a similar mixture, with largest weight for the $\mathrm{v} 3$ component, we will loosely refer to $v 3$ as the tau neutrino component.) Also required, however, is an enhanced probability for emission of $v 3$ near its low energy threshold. If this enhancement is due to new interactions between low energy $v 3$ neutrinos and nuclei, then the tritium $\beta$ decay experiments could be used as detectors for cosmic background neutrinos. Instead of tritium emitting a neutrino along with the electron, it could absorb a neutrino from the cosmic background [4]. There would then be a peak in the electron spectrum $\mathrm{m}_{\mathrm{v} 3}$ past the endpoint, $\mathrm{E}_{0}$. The phenomenological model we present for the $\beta$ decay spectrum anomaly also implies neutrinoless double $\beta$ decay may be observable by the current generation of experiments.

For $\mathrm{m}_{V} \neq 0$, the left handed electron neutrino is a mixture of three massive neutrino species [5],

$$
V_{e}=U_{e 1} V_{1}+U_{e 2} V_{2}+U_{e 3} V_{3}
$$

with

$$
\left|U_{e 1}\right|^{2}+\left|U_{e 2}\right|^{2}+\left|U_{e 3}\right|^{2}=1
$$

The electron neutrino is mostly $v_{1}$, with small admixture of the other two mass eigenstates. The muon and tau neutrinos are different mixtures of the same mass eigenstates, with the muon neutrino mostly $v_{2}$, and the tau neutrino mostly $v_{3}$. We assume neutrino oscillations explain the solar neutrino problem [6], implying,

$$
m_{V_{2}}^{2}-m_{V_{1}}^{2} \approx 10^{-5}
$$

We also assume the neutrino masses are determined by the see-saw mechanism [7],

$$
m_{V_{i}} \approx \frac{m_{q_{i}^{2 / 3}}^{2}}{M_{\text {Majorana }}} .
$$


relating the neutrino masses to the masses of charge $2 / 3$ quarks and a super heavy Majorana mass of order $10^{12} \mathrm{eV}$. Eqns. 3 and 4 with $\mathrm{m}_{\mathrm{c}} / \mathrm{m}_{\mathrm{u}} \sim 310^{2}$ imply $\mathrm{m}_{\mathrm{V} 1} \sim 310^{-8}$ $\mathrm{eV}$, and $\mathrm{m}_{\mathrm{V} 2} \sim 2-310^{-3} \mathrm{eV}$. Assuming $\mathrm{m}_{\mathrm{t}} / \mathrm{m}_{\mathrm{C}} \sim 100$, this suggests $\mathrm{m}_{\mathrm{V}} 3 \sim 20-30 \mathrm{eV}$. This is the correct scale associated with the observed anomalous behavior of the $\beta$ spectrum.

From Eq.(1), the spectrum measured in $\beta$ decay is a superposition of spectra. Most of the weight is associated with $v_{1}$ and $v_{2}$, which are essentially massless on the resolution scale of the experiments of a few $\mathrm{eV}$. The probability to emit $\mathrm{v} 3$ could be as large as a few $\%$ without violating other experimental limits [8]. We interpret the enhanced number of high energy electron events as due to an enhanced probability for the emission of low momentum v3. For electrons of energies $\mathrm{Ee}_{\mathrm{e}}<\mathrm{E}_{0}-\mathrm{m}_{\mathrm{v}} 3$, the spectrum is indistinguishable from a massless neutrino spectrum. For electron energies $\mathrm{E}_{0}-\mathrm{m}_{\mathrm{v}} 3<\mathrm{Ee}<\mathrm{E}_{0}$, the spectrum is essentially that of massless neutrinos but with weight $\left|U_{1}\right|^{2}+\left|U_{e}\right|^{2}=1-$ $\left|\mathrm{Ue}_{3}\right|^{2}$, that is, slightly less intense. In principle, Ue3 could be measured, but the rapid deterioration in statistics in the last $20 \mathrm{eV}$ makes such a measurement very difficult. In the region $\mathrm{Ee}>\sim \mathrm{E}_{0}-\mathrm{m}_{\vee} 3$, the enhanced probability to emit electrons is interpreted as an enhanced probability to emit low energy v3. Thus, in order to explain the $\beta$ spectrum, we must assume there is some final state interaction of $v_{3}$ that can enhance the probability to emit $v_{3}$ near its low energy threshold, analogous to the final state Coulomb interaction that enhances the spectrum of low momentum electrons.

The phase space contribution to the spectrum [9],

$$
P\left(E_{e}\right) \propto p_{e} E_{e} p_{v} E_{v},
$$

where $\mathrm{E}=\sqrt{ }\left(\mathrm{p}_{2}+\mathrm{m}_{2}\right)$, would vanish for $\mathrm{p}_{\mathrm{e}} \longrightarrow 0$, except for the Coulomb enhancement. This Coulomb enhancement is described by the Fermi function, the absolute square of the continuum Coulomb wavefunction near the origin, which nonrelativistically is,

$$
\begin{aligned}
& F\left(Z, p_{e}\right)=\frac{2 \pi Z \alpha}{\beta_{e}} /\left[1-\exp \left(-\frac{2 \pi Z \alpha}{\beta_{e}}\right)\right] \\
& \rightarrow\left\{\begin{array}{l}
1+\frac{\pi Z \alpha}{\beta_{e}}, \beta_{e} \rightarrow 1 \\
\frac{2 \pi Z \alpha}{\beta_{e}}, \beta_{e} \rightarrow 0
\end{array}\right.
\end{aligned}
$$


where $\beta_{e}=\left(p_{e} c\right) / E e . ~ A s ~ \beta_{e}>0$, the $1 / p_{e}$ Coulomb enhancement cancels a factor of $p_{e}$ in the phase space,

$$
P\left(E_{e}\right) F\left(Z, p_{e}\right) \sim 2 \pi Z \alpha E_{e}{ }^{2} p_{v} E_{v} \approx 2 \pi Z \alpha m_{e}{ }^{2} E_{0}{ }^{2}
$$

If we assume there is an analogous enhancement of the low momentum spectrum of v3, the $\beta$ spectrum becomes,

$$
\begin{aligned}
P\left(E_{e}\right) \longrightarrow & \left(\left|U_{e 1}\right|^{2}+\left|U_{e 2}\right|^{2}\right) P\left(E_{e}, m_{v} \approx 0\right) F\left(Z, p_{e}\right)+ \\
& \left|U_{e 3}\right|^{2} P\left(E_{e}, m_{v_{3}} \approx 23 e V\right) F\left(Z, p_{e}\right) F_{v}\left(p_{v}\right)
\end{aligned}
$$

If we assume $\left|\mathrm{Ue}_{3}\right|^{2} \sim 1 \%$, and if for very small $\mathrm{p}_{v 3}, \mathrm{~F}_{v}\left(\mathrm{p}_{v}\right) \sim \mathrm{A} / \mathrm{p}_{v}$, then $\mathrm{A}$ must be $\sim 30 \mathrm{eV} / \mathrm{c}$ in order to fit the $\beta$ spectrum. This very large value for $A$ will be very hard to understand. The other required feature of the enhancement, that only the low momentum $v_{3}$ spectrum should be enhanced, and not $v_{1}$ and $v_{2}$, will be much easier to make plausible. The electron contribution to the electron spectrum as $\mathrm{p}_{v} \rightarrow 0$ has the form,

$$
\left|U_{e 3}\right|^{2} P\left(E_{e}, m_{v_{3}}\right) F\left(Z, p_{e}\right) F_{v}\left(p_{v}\right) \sim .01 p_{e} E_{e} E_{v} A \theta\left(E_{m}-E_{e}\right)
$$

where the theta function in the above equation shows the electron spectrum for emission of $v_{3}$ ends at $E_{m}=E_{0}-m_{v} 3$. The electron spectrum does not vanish as $p_{v} 3->0$, but forms a ledge of height proportional to

$$
.01 \sqrt{2 m_{e} E_{m}}\left(m_{e}+E_{m}\right) m_{v 3}^{2} A
$$

at $E_{\mathrm{e}}=\mathrm{E}_{0}-\mathrm{m}_{\mathrm{V}}$. The spectrum from $\mathrm{E}_{\mathrm{m}}$ to $\mathrm{E}_{0}$ is that of essentially massless neutrinos, but with only $99 \%$ of the weight of the massless spectrum far below $E_{m}$. One could in principle measure $\left|\mathrm{Ue}_{3}\right|^{2}$ from the relative intensity of the massless neutrino spectrum for $\mathrm{E}_{\mathrm{e}}<<\mathrm{E}^{\mathrm{m}}$ and for Ee between $\mathrm{E}_{0}-\mathrm{m}_{\mathrm{v}} 3$ and $\mathrm{E}_{0}$.

What could be responsible for this final state interaction? By analogy with the Coulomb enhancement of the low energy electron spectrum, we need an attractive interaction between the ${ }^{3} \mathrm{He}$ nucleus and $\mathrm{v} 3$ that is trying to make a bound state. Such an interaction will then create an instability in the perturbation theory. $\mathrm{Z}$ bosons are the first candidate. Ordinary weak interaction radiative corrections have previously been calculated assuming massless neutrinos [10]. The effects of neutrino mass, though, give non leading contributions to the weak interaction radiative corrections [11]. There are, 
however, now Higgs boson interactions for massive neutrinos, since the neutrinos have Dirac masses. Also, since the Dirac mass for $v_{3} \sim m_{t}$, there could possibly be very strong Higgs interactions. Since the Higgs boson is so heavy, even if the interaction were strong, there would be little effect [12]. We need a lighter particle.

Massive neutrinos can interact with the ${ }^{3} \mathrm{He}$ nucleus through neutral pi mesons. This statement is less shocking if one considers $\pi^{0}$ decay to neutrino pairs [13]. The interaction between neutral pions and massive neutrinos is because the pion in the standard model is actually a mixture of the QCD pion and the Higgs fields [14]. The chiral field,

$$
\begin{aligned}
& U(x)=\sigma+i \pi \cdot \tau=\left(\begin{array}{cc}
\sigma+i \pi^{3} & i\left(\pi^{1}-i \pi^{2}\right) \\
i\left(\pi^{1}+i \pi^{2}\right) & \sigma-i \pi^{3}
\end{array}\right)= \\
& {\left[\left(\begin{array}{c}
\sigma+i \pi^{3} \\
i\left(\pi^{1}+i \pi^{2}\right)
\end{array}\right)\left(\begin{array}{c}
i\left(\pi^{1}-i \pi^{2}\right) \\
\sigma-i \pi^{3}
\end{array}\right)\right],}
\end{aligned}
$$

is a complex spinor and the charge conjugate spinor. Gauging the $S U(2) L$ symmetry in the chiral Lagrangian shows that with $\langle\sigma\rangle=\mathrm{f}_{\pi}$ the $\mathrm{W}$ and $\mathrm{Z}$ become massive with mass gf $_{\pi}[15]$. There is no pion, only massive $W$ and $Z$ fields. The spinor in the chiral field has the same form as the usual Higgs complex spinor,

$$
\left(\begin{array}{c}
i\left(\phi^{1}-i \phi^{2}\right) \\
h-i \phi^{3}
\end{array}\right)
$$

and therefore they mix. One linear combination of the QCD pion and the Higgs $f$ supply the degrees of freedom needed for the third polarization states of the massive spin $1 \mathrm{~W}$ and $\mathrm{Z}$ particles; the other orthogonal linear combination is the physical pion,

$$
\pi_{\text {physical }} \sim i \bar{q} \gamma_{5} \tau q+\frac{f_{\pi}}{v} \phi
$$

where $v=\langle h\rangle$, is the usual Higgs field vacuum expectation value. The physical pions therefore couple to all particles that the Higgs couples to; For neutrinos with electroweak scale Dirac mass terms, the neutral pions couple directly. If the Dirac mass term for the neutrinos is proportional to the charge $2 / 3$ quark mass terms, then the $\pi^{0}$ couples to $v 3$ proportional to the top quark mass; the $v_{2}$ coupling is 100 times weaker, and the $v_{1}$ 
coupling is 300 times weaker still. The nucleon-v3 interaction strength through the neutral pion would be

$$
\begin{aligned}
& \mathrm{g}_{\pi N \mathrm{~N}} \frac{\mathrm{f}_{\pi}}{\mathrm{v}} \frac{\mathrm{m}_{\mathrm{t}}}{\mathrm{v}} \sim \mathrm{g}_{\pi \mathrm{NN}} \mathrm{f}_{\pi} \mathrm{G}_{\mathrm{F}} \mathrm{m}_{\mathrm{t}}= \\
& 2 \mathrm{~g}_{\mathrm{A}} \mathrm{MG} \mathrm{G}_{\mathrm{t}} \sim 2.510^{-5} 150 \sim 410^{-3}
\end{aligned}
$$

that is, a strength comparable to the electromagnetic interaction, $\sim 7.310^{-3}$. This result follows from the large Dirac mass term of $v 3$. Unfortunately, this is not the correct answer. We have not yet included the Majorana mass term,

$$
M_{\text {Majorana }} \overline{V_{L}^{c}} V_{R}
$$

where the charge conjugate of the right handed neutrino is left handed. In constructing mass eigenstates for the neutrinos they become Majorana particles, the left handed particles being very light, and the right handed fields super massive [5]. The diagonalization of the neutrino mass matrix, requiring a rotation of the basis, introduces a mixing angle into the $\pi^{0}$ coupling,

$$
\sin (2 \theta) \sim \mathrm{m}_{1} / \mathrm{M}_{\text {Majorana }}
$$

that suppresses the $\pi^{0}-v 3$ coupling, turning the top quark mass into the neutrino mass, a suppression factor of $10^{-10}$. (Another way to see this factor is to consider $\pi^{0}$ decay to neutrino pairs. The spin zero particle decays to a very light left handed neutrino and, to conserve angular momentum, a left handed antineutrino. The left handed antineutrino is super massive, but has a small mixture, of order $\mathrm{m}_{\mathrm{t}} / \mathrm{M}_{\mathrm{Majorana}}$, of the light neutrino.)

Despite the small coupling, one could still ask about whether the $\pi^{0}$ exchange final state interaction between the ${ }^{3} \mathrm{He}$ and $v_{3}$ could produce an enhancement of the low momentum v3 spectrum. After all, the pions can produce bound states of nucleons and anti nucleons as well as nucleons and nucleons. The physics of the bound state formation can be seen from the perturbation theory Feynman diagram for single particle exchange, analogous to the QED radiative correction for the final state electron - nucleon interaction [16]. The physics responsible for the Coulomb enhancement is made most clear from a dispersion relation treatment of the Feynman diagram [17]. A particle pair is emitted in a virtual state of center of mass energy $M$, and that pair then scatters through the exchanged 
particle. The Coulomb enhancement arises from the forward scattering singularity at zero momentum transfer. One obtains,

$$
\mathrm{m}_{\pi}^{2} \int_{\left(\mathrm{M}_{\mathrm{n}}+\mathrm{m}_{\mathrm{V}}\right)^{2}}^{\infty} \frac{\mathrm{d} \mathrm{M}^{2}}{\mathrm{k}^{2}-\mathrm{M}^{2}} \frac{1}{2 \mathrm{Mp}} \ln \left(\frac{4 \mathrm{p}^{2}}{\mathrm{~m}_{\pi}^{2}}+1\right)
$$

where the log arises from the angular average of the reciprocal of the momentum transfer,

$$
\int_{-1}^{1} \frac{d \cos (\theta)}{2} \frac{1}{-2 p^{2}(1-\cos (\theta))-\mu^{2}}=-\frac{1}{2 p^{2}} \ln \left(\frac{4 p^{2}}{\mu^{2}}+1\right)
$$

and where

$$
\mathrm{p}=\frac{\mathrm{M}}{2} \sqrt{1-\frac{\left(\mathrm{M}_{\mathrm{n}}+\mathrm{m}_{V}\right)^{2}}{\mathrm{M}^{2}}} \sqrt{1-\frac{\left(\mathrm{M}_{\mathrm{n}}-\mathrm{m}_{\mathrm{V}}\right)^{2}}{\mathrm{M}^{2}}}
$$

is the common magnitude of momentum of the neutrino and nucleon in the center of mass frame of the decay of a virtual mass, $M=\sqrt{ }\left(p^{2}+m_{v}{ }^{2}\right)+\sqrt{ }\left(p^{2}+M_{n}{ }^{2}\right)$, and $k^{2}=\left(p_{v}+p_{N}\right)^{2}$ is the invariant total center of mass energy squared of the neutrino and nucleon. In the analog QED case, in which the nucleon and electron are interacting through a photon, the pion mass in the log is replaced by an infinitesimal infrared cutoff, making the 1 term in the $\log$ unimportant. In that limit, the spectral integral $\sim 1 / \mathrm{v}$, where $\mathrm{v}$ is the magnitude of the relative velocity of the two particles. (Also the coefficient in front of the integral is an invariant energy, not the photon mass [16].) Here, the pion mass cuts off the forward scattering singularity. Since the dominant momentum is small compared to the pion mass, there is no enhancement from the log, and so no analog of the Coulomb enhancement.

In order to get an enhancement, we must consider the exchange of a particle much lighter than the neutrino. A candidate is the axion, which behaves like a light neutral pion of mass of perhaps $\sim 10-5 \mathrm{eV}$. Like the Higgs particles, the axion coupling to fermions is proportional to the fermion mass, so the same hierarchy of strength of coupling to the three neutrino mass eigenstates found for the pion interaction would also apply to the neutrino interaction. Of course the axion couplings are weak compared to the pion, but it nevertheless gives a qualitative enhancement.

Conclusions: 
The observed tritium $\beta$ decay spectrum is usually fit to negative (mass) ${ }^{2}$. We have shown there is a consistent phenomenological interpretation of the data due to a positive mass tau neutrino (we refer to $v 3$, which has a small amplitude in the electron neutrino, but very large component in the tau neutrino, as a tau neutrino) component to ordinary $\beta$ decay with strong near threshold (for the low energy neutrino) final state interactions with the He nucleus. The model predicts a distinctive spectrum shape that is consistent with the latest high resolution data [2]. The data suggest mv3 $\sim 23 \mathrm{eV}$. Such a mass would imply that tau neutrinos dominate the mass of the universe $[4,5]$. This model for the tritium $\beta$ decay anomaly implies neutrinoless double $\beta$ decay could be observable by the current generation of experiments [18]. This is because the neutrinos in this model are Majorana particles, and the effective neutrino mass in double $\beta$ decay would be $\left.\lambda e 3\right|^{2}$ $\mathrm{m}_{\mathrm{V}} 3 \sim 4 \% 23 \mathrm{eV} \sim 1 \mathrm{eV}$. (The $4 \%$ branching probability is the largest allowed by accelerator neutrino oscillation experiments [8].)

Theoretically, the modifications of weak interaction radiative corrections to $\beta$ decay due to assumed neutrino masses are negligible [11]. In order to realize the phenomenological mechanism from fundamental new physics, one needs, first, a final state interaction between neutrinos and nuclei that is strong for $v_{3}$, and negligibly weak for $v_{2}$ and especially $v_{1}$. We have found two interactions that satisfy this requirement, one through neutral pions (perhaps the most interesting and surprising result of this study), and the other through axions. One further needs an interaction that can produce a factor of $1 / \mathrm{p} v 3$ correction to the $\beta$ decay spectrum, that would enhance the probability to emit low energy v3, and correspondingly high energy electrons with $\mathrm{E}_{\mathrm{e}}$ just below $\mathrm{E}_{0}$ mv3. We have found that the axion could produce such a Coulomb-like enhancement factor. However, the axion couplings are infinitesimal compared to required strengths, and also the mass dependence of its coupling to neutrinos would cancel. The axion, being a pseudoscalar particle, actually couples to spin at low energy. The mass dependence of the pseudoscalar coupling is absorbed into a momentum times spin coupling. A very light scalar particle, like a very light Higgs particle, which could also have couplings proportional to mass (as the very heavy Higgs particle does), could produce a neutrino mass dependent enhancement. However, the coupling strength required would dominate gravity at distances $\ll 1 \mathrm{~cm}$.

We have examined some possible fundamental physics explanations for the observed tritium $\beta$ decay spectrum anomaly. Models of elementary particle physics that predict neutrino mass arise from attempts to unify the electroweak and strong 
interactions. We have examined some of the implications of such models. We have shown that the parameters required of such models are much too large to be considered plausible. We therefore expect that the explanation for the tritium $\beta$ decay spectrum anomaly lies in the molecular physics. The branching probabilities to the various bound, resonant, and continuum final states of the $\mathrm{T}-{ }^{3} \mathrm{He}$ molecular ion are assumed in the data analysis. The $\beta$ decay spectrum is a superposition of spectra associated with the possible molecular final states. These molecular branching probabilities should be re-examined in light of these results.

\section{Acknowledgements:}

I wish to thank Wolfgang Stoeffl, Dan Decman, Steve Libby for many valuable discussions. This work was performed by LLNL under the auspices of the U. S. Department of Energy under contract No. W-7405-Eng-48. 
Appendix: Technical details of the enhancement integral

In order to evaluate the spectral integral, $\mathrm{M}^{2}$ in Eqs.(17) and (19) is expressed in terms of the velocities of the neutrino and nucleon,

$$
M^{2}=M_{n}^{2}+m_{v}^{2}+2 M_{n} m_{v} \frac{\left(1+V_{n} V_{v}\right)}{\sqrt{1-V_{n}^{2}} \sqrt{1-V_{v}^{2}}}
$$

and the relative velocity is introduced,

$$
\mathrm{v}=\frac{\mathrm{V}_{\mathrm{n}}+\mathrm{V}_{\mathrm{v}}}{1+\mathrm{V}_{\mathrm{n}} \mathrm{V}_{\mathrm{v}}}
$$

and the relation

$$
1-v^{2}=\frac{\left(1-v_{n}^{2}\right)\left(1-v_{v}^{2}\right)}{\left(1+v_{n} v_{v}\right)^{2}}
$$

Then in terms of $v$,

$$
M^{2}=M_{n}^{2}+m_{v}^{2}+\frac{2 M_{n} m_{v}}{\sqrt{1-v^{2}}}
$$

and

$$
\mathrm{M}_{\mathrm{n}}^{2} \mathrm{~m}_{\mathrm{v}}^{2} \frac{\mathrm{v}^{2}}{\sqrt{1-\mathrm{v}^{2}}}=\mathrm{p}^{2} \mathrm{M}^{2}
$$

With the additional change of variables,

$$
1-v^{2}=\left(\frac{1-u^{2}}{1+u^{2}}\right)^{2}
$$

we have, 


$$
M^{2}=\frac{\left(M_{n}+m_{v}\right)^{2}-\left(M_{n}-m_{v}\right)^{2} u^{2}}{1-u^{2}}
$$

and

$$
M_{n}^{2} m_{v}^{2} \frac{u^{2}}{\left(1-u^{2}\right)^{2}}=p^{2}\left[\left(M_{n}+m_{v}\right)^{2}-\left(M_{n}-m_{v}\right)^{2} u^{2}\right]
$$

The spectral integral then becomes,

$$
\begin{aligned}
& \int_{\left(M_{n}+m_{v}\right)^{2}}^{\infty} \frac{d M^{2}}{k^{2}-M^{2}} \frac{1}{2 M_{p}} \ln \left(\frac{4 p^{2}}{m_{a}^{2}}\right)= \\
& \int_{0}^{1} \frac{d u^{\prime 2}}{u^{\prime 2}} \frac{\left[\frac{16 M_{n}^{2} m_{v}^{2}}{m_{a}^{2}} \frac{u^{\prime 2}}{\left(1-u^{\prime 2}\right)^{2}} \frac{1}{\left(M_{n}+m_{v}\right)^{2}-\left(M_{n}-m_{v}\right)^{2} u^{\prime 2}}\right]}{\left(1-u^{\prime 2}\right) k^{2}-\left(M_{n}+m_{v}\right)^{2}+\left(M_{n}-m_{v}\right)^{2} u^{\prime 2}},
\end{aligned}
$$

and with the substitution,

$$
\mathrm{k}^{2}=\frac{\left(\mathrm{M}_{\mathrm{n}}+\mathrm{m}_{\mathrm{v}}\right)^{2}-\left(\mathrm{M}_{\mathrm{n}}-\mathrm{m}_{\mathrm{v}}\right)^{2} \mathrm{u}^{2}}{1-\mathrm{u}^{2}}
$$

becomes,

$\frac{1-u^{2}}{2 M_{n} m_{v}} \int_{0}^{1} \frac{d u^{\prime}}{u^{2}-u^{\prime 2}} \ln \left[\frac{16 M_{n}^{2} m_{v}^{2}}{m_{a}^{2}} \frac{u^{\prime 2}}{\left(1-u^{\prime 2}\right)^{2}} \frac{1}{\left(M_{n}+m_{v}\right)^{2}-\left(M_{n}-m_{v}\right)^{2} u^{\prime 2}}\right]$

With the quadratic form in both the denominator and the log, this integral can now be factored and integrated to Spense functions plus the required $\pi^{2} / \mathrm{v}$ term. The remaining analysis follows Schwinger' $s$ treatment of the radiative corrections to vacuum polarization [17]. 


\section{References}

1. R. G. H. Robertson, et. al., Phys. Rev. Lett. 67957 (1991); H. Kawakami, et. al. Phys. Lett. 256B 105 (1991); M. Fritschi, E. Holzschuh, and W. Kuendig, Phys. Lett. 287B 381 (1992); Ch. Weinheimer, et. al., Phys. Lett. 300B 210 (1993); W. Stoeffl and D. Decman, "Anomalous structure in the beta decay of gaseous molecular tritium," UCRL 3C-115771 (submitted to Phys. Rev. Lett.).

2. W. Stoeffl and D. Decman, Ref. [1].

3. R. S. VanDyck, D. L. Farnham, and P. B. Schwinberg, Phys. Rev. Lett. 702888 (1993).

4. J. M. Irvine and R. Humphrieys, J. Phys. G 9847 (1983); S. Weinberg, Gravitation and Cosmology, (John Wiley and Sons, New York, 1972).

5. For a review, see, for example, B. Kayser, Physics of Massive Neutrinos, (World Scientific, Teaneck, NJ, 1989); T. P. Cheng and L.-F. Li, Gauge Theory of Elementary Particle Physics, (Clarendon Press, Oxford, 1984).

6. J. N. Behcall and H. A. Bethe, Phys. Rev. Lett. 652233 (1990); For a review of the Mikheyev-Smirnov-Wolfenstein effect, see, for example, T. K. Kuo and J. Pantaleone, Rev. Mod. Phys. 61937 (1989).

7. M. Gell-Mann, P. Ramond, and R. Slansky, in Supergravity, edited by D. Z. Freedman and P. Van Niewenhuizen (North-Holland, Amsterdam,1979).

8. A. V. Manohar and A. E. Nelson, Phys. Rev. Lett. 662847 (1991).

9. D. H. Wilkinson, Nucl. Phys. A 526131 (1991).

10. A. Sirlin, Rev. Mod. Phys. 50573 (1978).

11. An enhancement of the high energy electron beta spectrum arising from small deviations from V-A for massive neutrinos was proposed by J. J. Sakurai, Phys. Rev. Lett. 140 (1958). Electroweak radiative corrections with massive neutrinos induce such form factor terms, but the leading contributions vanish, making these terms quantitatively neglegible, as they are proportional to powers of $\mathrm{m}_{\mathrm{V}} / \mathrm{MW}$. (N. J. Snyderman, unpublished). For a general discussion and experimental limits on measuring small induced form factors, see D. H. Wilkinson, Nucl. Phys. A 377474 (1982). 
12. This can be seen using same kind of analysis in M. K. Gaillard and B. W. Lee, Phys. Rev. D 10897 (1974), and in A. Sirlin, Ref. [10].

13. B. Kayser et. al., Phys. Lett. 52 B 385 (1974); P. Herczeg and C. M. Hoffman, Phys. Lett. 100 B 347 (1981).

14. M. Weinstein, Phys. Rev. D7 1854 (1973).

15. S. Weinberg, Phys. Rev. D 191277 (1979); L. Susskind, Phys. Rev. D 202619 (1979).

16. R. E. Behrends, R. J. Finkelstein, and A. Sirlin, Phys. Rev. 101866 (1956); S. M. Berman, Phys. Rev. 112267 (1959); T. Kinoshita and A. Sirlin, Phys. Rev. 1131652 (1959); D. Bailin, Phys. Rev. 135 B166 (1964).

17. J. Schwinger, Particles Sources and Fields II, (Addison-Wesley, Reading MA, 1973).

18. F. T. Avignone III, et. al., Phys. Rev. C 34666 (1986). 